\title{
Relationships and the social brain: Integrating psychological and evolutionary perspectives
}

\author{
Alistair Sutcliffe ${ }^{1 *}$, Robin Dunbar², Jens Binder ${ }^{1,3}$ \\ and Holly Arrow ${ }^{4}$ \\ 'Manchester Business School, University of Manchester, UK \\ ${ }^{2}$ British Academy Centenary Research Project, Institute for Cognitive \& \\ Evolutionary, Anthropology, University of Oxford, UK \\ ${ }^{3}$ Division of Psychology, School of Social Sciences, Nottingham Trent University, \\ Nottingham, UK \\ ${ }^{4}$ Department of Psychology \& Institute of Cognitive and Decision Sciences, \\ University of Oregon, Portland, Oregon, USA
}

\begin{abstract}
Psychological studies of relationships tend to focus on specific types of close personal relationships (romantic, parent-offspring, friendship) and examine characteristics of both the individuals and the dyad. This paper looks more broadly at the wider range of relationships that constitute an individual's personal social world. Recent work on the composition of personal social networks suggests that they consist of a series of layers that differ in the quality and quantity of relationships involved. Each layer increases relationship numbers by an approximate multiple of 3 (5-I5-50-150) but decreasing levels of intimacy (strong, medium, and weak ties) and frequency of interaction. To account for these regularities, we draw on both social and evolutionary psychology to argue that relationships at different layers serve different functions and have different cost-benefit profiles. At each layer, the benefits are asymptotic but the costs of maintaining a relationship at that level (most obviously, the time that has to be invested in servicing it) are roughly linear with the number of relationships. The trade-off between costs and benefits at a given level, and across the different types of demands and resources typical of different levels, gives rise to a distribution of social effort that generates and maintains a hierarchy of layered sets of relationships within social networks. We suggest that, psychologically, these trade-offs are related to the level of trust in a relationship, and that this is itself a function of the time invested in the relationship.
\end{abstract}

Social relationships are studied in numerous fields of basic and applied psychology. Within social psychology, relationships are a fundamental component in models of

\footnotetext{
* Correspondence should be addressed to Alistair Sutcliffe, Manchester Business School, University of Manchester, Booth Street West, Manchester MI5 6PB, UK (e-mail: Alistair.Sutcliffe@mbs.ac.uk).
} 
social exchange (Kelley et al., 2003; Moreland \& Levine, 1982; Rusbult \& Van Lange, 2003) and affiliation (Baumeister \& Leary, 1995), as well as in theories of the relational self (Andersen \& Chen, 2002; Brewer \& Gardner, 1996; Markus \& Kitayama, 1991). Within health psychology and the literature on subjective well-being, relationships are both a source of social support (Cohen \& Wills, 1985; Golden, Conroy, \& Lawlor, 2009; Seeman, Lusignolo, Albert, \& Berkman, 2001) and a determinant of quality of life (Cohen, 2004; Helgeson, 2003). In studies of internet use, the transformative impact of digital technology on relationship maintenance (Ellison, Steinfield, \& Lampe, 2007; Kraut et al., 2002; Pollett, Roberts, \& Dunbar, 2011) and relationship formation (Mesch \& Talmud, 2007; Morahan-Martin \& Schumacher, 2003) are hotly debated. This list could easily be expanded.

Across these diverse literatures, most studies focus either on intra-individual processes such as social perception and the self or on the properties of dyads, without locating these processes within a broader social context. In this paper, we step back and take a wider view, drawing on theory from evolutionary psychology that connects the individual level to the broader ecology of groups and networks in which social interaction unfolds. We also propose a model that integrates the evolutionary viewpoint with cognitive mechanisms of trust that might account for observed, existing social structures, while being grounded in a parsimonious set of behavioural predispositions produced by natural selection. While evolutionary psychology is best known for emphasizing the fitness benefits of relationships (e.g., Brown \& Brown, 2006), it has also introduced the idea that the costs and benefits of social interactions are a critical driver for cognitive evolution.

In this paper, we integrate an evolutionary perspective with a psychological perspective to account for some intriguing regularities that recent work has documented in the layered structure of personal social networks. We hope to spark interest among the many psychologists who study relationships to help explain why it is that social networks appear to consist of a set of distinct layers of relationships that differ in both quantity and quality. We believe this project fits with Bersheid's (1994) plea for more interdisciplinary work to help integrate research on different kinds of relationships. We first identify the phenomenon to be explained: both egocentric social networks and social groups appear to be structured in hierarchically organized layers that scale consistently in characteristic sizes. Second, we offer a principled explanation for how and why such tiering of social relationships might be expected to occur. In doing so, we build on and extend Brown \& Brown's (2006) evolutionarily motivated analysis of close relationships. Finally, we relate this tiering to the role of trust in the maintenance of dyadic social relationships.

\section{Relationships, group size, and the structure of social networks}

We begin this section with a brief summary of primate sociality, since it provides the crucial context for an evolutionary perspective on human sociality. Primates in general (and humans in particular) live in social groups that have long-term stability and coherence, even where (as in chimpanzees and humans) these groups are spatially dispersed. Such groups are an emergent property of individual dyadic relationships (Dunbar \& Shultz, 2010). For any given population, group size (the functional outcome of sociality) appears to be optimized by balancing the benefits against the costs of group living. For primates as with most mammals, the primary evolutionary driver providing the benefits of group living has been predation risk (Shultz, Noe, McGraw, \& Dunbar, 2004; van Schaik, 1983), even though other secondary benefits (e.g., territory defence, 
alloparenting) may have arisen as secondary benefits once group living was in place (Dunbar, 1988); the costs are reflected in the negative effects of ecological and social competition, all of which are likely to be group-size dependent. For any given species, the typical balance between costs and benefits defines an average group size that sets the norm for that species.

Primate social groups are, in effect, implicit social contracts in which individuals cooperate to share the long-term costs of minimizing predation risk, while incurring short-term costs in respect of immediate personal interests (Dunbar, 2008). Since maintaining coherent groups is cognitively demanding, brain size (or more specifically neocortex volume) will evolve to match the cognitive demands of the species' optimal group size. This claim forms the core of what has become known as the social brain hypothesis (SBH) (Dunbar, 1992, 1998a; Dunbar \& Shultz, 2007; Shultz \& Dunbar, 2007,2010 ). Though widely discussed within biology and evolutionary anthropology as a plausible explanation linking social and cognitive evolution, the $\mathrm{SBH}$ is relatively unknown within psychology, despite its profound implications for a wide range of social relationships

The formation of cohesive social groups of the kind found in primates inevitably creates costs because individuals are forced to forage and rest in close proximity, and are unable to diffuse within-group aggression easily by distancing themselves from those with whom they are in conflict. If these tensions are not adequately resolved, conflict will split groups apart and the benefits of grouping will be lost. Primates buffer themselves against these costs by forming small coalitions of two or three individuals that reduce the frequency of aggression or harassment (Dunbar, 2010a). In sum, for primates, grouping to reduce predation risk creates internal costs, which are in turn resolved by further micro-scale groupings within the community as a whole. As a result, social groups naturally consist of sets of lower level groupings nested within a larger grouping, with some species effecting as many as four or five layers, much as we find in humans (Hill, Bentley, \& Dunbar, 2008; Zhou, Sornette, Hill, \& Dunbar, 2005). Note that although predation risk provides the principal explanation for the evolution of group size in primates as a whole, this cannot be true for the handful of species (notably chimpanzees and humans) that live in so-called fission-fusion social systems where the group (or community) does not forage as a single unit. In these cases, the anti-predation function is linked with the foraging group (one of the inner layers) and the main SBH-predicted outer grouping layer has instead become associated with other functions that have yet to be conclusively identified (possibilities include territory defence or access to mates).

Applied to humans, the primate $\mathrm{SBH}$ relationship predicts an average group (i.e., community) size of around 150, a value that has been documented in a wide range of social, cultural, and historical circumstances, representing both top-down (community level) and bottom-up (egocentric personal network) perspectives (Dunbar, 1993; Hamilton, Milne, Walker, Burger, \& Brown, 2007; Hill \& Dunbar, 2003; Roberts, Dunbar, Pollet, \& Kuppens, 2009; Zhou et al., 2005). Examples include community size in traditional hunter-gatherers, average countywide village sizes in both the Domesday Book and eighteenth century England, company size in historical and modern armies, and parish sizes among the Amish and Huterites (Dunbar, 2008). This convergence between whole group size and egocentric network size is striking, given that they are clearly not the same thing. The fact that these two perspectives yield the same numbers is thus surprising, and remains unexplained.

Viewed from the perspective of egocentric social networks, 150 represents the active network of individuals that we know as persons and with whom we have reciprocated, 


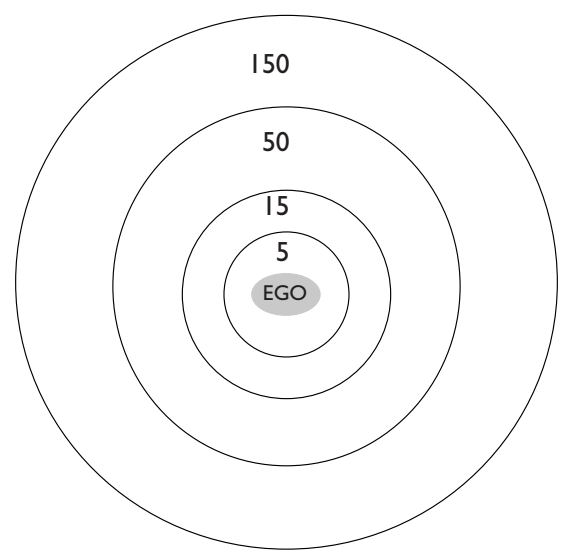

Figure I. Schematic illustration of the layering of relationships in social networks. EGO indicates the individual whose personal network is being illustrated. The layers correspond to the hierarchically inclusive number of other individuals ('Alters') who are members of Ego's social network (after Roberts, 2010).

personalized relationships that have a history (we know how they fit into our social world and they know how we fit into theirs). As is the case in primates as a whole, this group of 150 is not homogenous in terms of the structure of relationships. Within the network as a whole, there are a series of 'circles of acquaintanceship' that seem to scale with a consistent ratio close to 3 (Hill \& Dunbar, 2003; Zhou et al., 2005; see also Hamilton et al., 2007). These layers (which are hierarchically inclusive) lie at around 5 , 15, 50, and 150 individuals (shown schematically in Figure 1). This layering pattern holds for both the bottom-up structure of egocentric networks and the top-down structure of communities and their constituent subgroups (something that is especially clear, e.g., in the structure of military units: Dunbar, 2010b).

In humans, the innermost two layers have been identified as the support clique of 4-5 and the sympathy group of 12-15 (Dunbar \& Spoors, 1995; Stiller \& Dunbar, 2007), the latter being already familiar from social psychology (Buys \& Larson, 1979). These are followed by the affinity group (or band in the ethnographic literature) of around 50 and the active network (clan in the ethnographic literature) of 150 individuals. For personal networks, these layers have been shown to represent natural disjunctions in both the level of intimacy between Ego and Alter $^{1}$ (Figure 2) and the frequency with which they interact (Hill \& Dunbar, 2003; Roberts et al., 2009), although, as yet, we do not know why social relationships should form these natural hierarchies or why the layers of an egocentric network should scale the same way that hierarchically nested group sizes scale.

This layering has implications for the patterning of relationships. Although close friends of a given individual are more likely to be friends (a property known as clustering or network transitivity), the probability that any two of my friends are also friends with each other tends to fall between $10 \%$ and $50 \%$ (Girvan \& Newman, 2002). So, the personal social networks of friends who both belong to the same large group of 150 overlap at best incompletely, even though each has an active network of about the same size.

\footnotetext{
'We use these terms as they are commonly used in the social network literature. Ego refers to the subject (or 'owner') of a personal network and Alter refers to the individual members of Ego's personal network.
} 


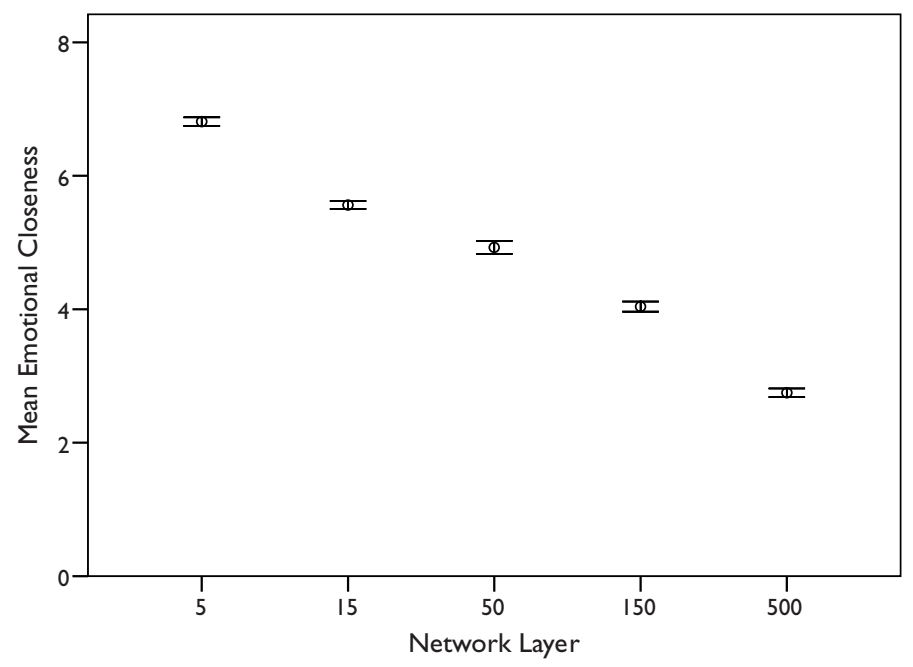

Figure 2. Mean $( \pm S E)$ in emotional closeness (indexed on a $1-10$ analogue scale) for Alters in each layer of the network for 295 women sampled in United Kingdom and Belgium. Network layers are defined by Alters ordered as $1-5,6-15,16-50,51-150$, and $151-500$ by time to last contact. Emotional closeness differs significantly across the layers $\left(F_{4,18538}=2002.4 ; p<.000 \mathrm{I}\right)$, with each layer differing significantly from all the others (Scheffé tests: $p<.001$ in each case). Sample sizes ( $L$ to $R$ ): 4770, 5209, 1934, 3288, 3342 Alters. Source: Roberts et al. (2009).

Although the broader pattern has not attracted much attention, there is considerable support in the literature for at least the two inner layers. Despite differences in the way close and not-so-close relationships are defined (Fischer, 1982; Grossetti, 2005; Milardo, 1992), studies of friendships and other human social relationships report broadly comparable network sizes based on this distinction. Using an intimate (socially close) versus routine (actively maintained) classification, Wellman, Carrington, and Hall (1988) found a mean of 5.0 intimate ties and 12.5 active ties, and reported that intimate relationships formed dense clusters, with active ties forming inter-cluster bridges. These values are in close agreement with those obtained by Dunbar and Spoors (1995) who used criteria based on both social support (advice and help at the personal level) and frequency of contact (weekly vs. monthly). In contrast, a wider ranging survey that used 'very close' and 'somewhat close' ties as criteria reported means of 11.6 and 16.8 ties for each level (Kayahara, Wellman, Boase, Hogan, \& Kennedy, 2005).

Name-generation studies of relationships, which elicit stronger ties by using a number of questions as cues give a fairly consistent mean network size of approximately 15 individuals (Fischer, 1982; Grossetti, 2007). Several studies on friendships have distinguished different levels of relationship intimacy without focusing explicitly on network structure. Hays (1989) compared close and casual friendships and found that close friendships showed more interactions during the week, across a wider range of days, times, and locations than casual friendships. In terms of benefits received, close friends offered more emotional and informational support than casual ones. Indeed, the most commonly used classification in these studies is best, close, and casual friend (Oswald, Clark, \& Kelly, 2004; Rose \& Serafica, 1986). Oswald et al. (2004) assessed friendship in terms of different dimensions of friendship maintenance behaviours (positivity, supportiveness, openness, and interaction), and found a clear 
distinction between higher scoring best friends on the one hand and lower scoring close and casual friends on the other. Rose and Serafica (1986) found that casual friends required less emotional involvement than best or close friends, but were more dependent on opportunities for contact. In contrast, best friendships were seen as being self-maintained.

In sum, consistent behaviour differences have been reported for different levels of relationship intensity. Friendships are usually seen as involving positive emotion, intimacy, support, and interaction (Brown \& Brown, 2006; Dainton, Zelley, \& Langan, 2003), and these psychological aspects are more pronounced in stronger friendships with best, close, and casual being a useful, although under-defined, classification. Studies of human relationships, therefore, generally support the hypothesis that we maintain different kinds of relationship, with a smaller number of intimate friendships. By and large, the focus of the social psychological literature has been on close or intimate relationships such as parent-offspring, romantic, and intimate friends relationships (Berscheid, 1994; Kelley et al., 1983; see also Brown \& Brown, 2006). However, given that our individual social worlds seem to consist of hierarchically layered sets of relationships, our task is to find a principled explanation for why such structures might exist.

One plausible explanation for the layering of relationships in human personal social networks is that the layers (i.e., the different relationship types) are designed to deal with different kinds of functions and/or constraints. For practical convenience, we restrict our remarks here to the two innermost layers (the 5 and 15) and the outermost (the 150). In his 'weak links' hypothesis, Granovetter (1973, 1985) proposed that information exchange is the function for the active egocentric network (in our schema, synonymous with the 150 layer). However, alternative and perhaps ethnographically more plausible possibilities are that larger networks serve functions of resource buffering (controlling access to foraging areas or creating a network of trading relationships) or community-level defence against neighbouring human groups (Alexander, 1974; Dunbar, 1996). In contrast, the innermost network layers of 5 and 15 are most likely to involve social support, which can be either mutual (friends) or directional (e.g., parent-offspring). They seem to represent the two levels of intimates on whom we really depend (and/or who depend on us) for facilitating (in a psychological sense) our pathway through the complex social world in which we live, with such relationships being mediated by the emotional basis of close friendships in just the way suggested by Brown \& Brown (2006) in their SIT ('selective investment theory') hypothesis. In terms of size, the sympathy group (typically 12-15 members) seems to correspond proportionately to the coalitions found in monkeys and apes (Kudo \& Dunbar, 2001), and in humans this may represent the group of reliable friends on whom one can depend for a variety of exchange relationships (e.g., friendship in the social sense, protection against harassment, minimizing social stress, distributed childcare, etc.). In contrast, the innermost layer of about 5, the support clique, seems to represent the set of closest intimates, typically immediate family members and best friends, who are most likely to provide a mutual environment for emotional and instrumental (e.g., financial) support.

A likely reason for this apparent partitioning of social relationships is that they pose contrasting trade-offs between the benefits and costs of maintaining relationships of different quality. In the following section, we discuss the constraints on the distribution of a social time budget that might lead to a mix of relationship types of this kind. Our account builds on the ideas developed by Brown \& Brown (2006) in the SIT hypothesis, but adds to this by setting it within a broader community-level perspective. 


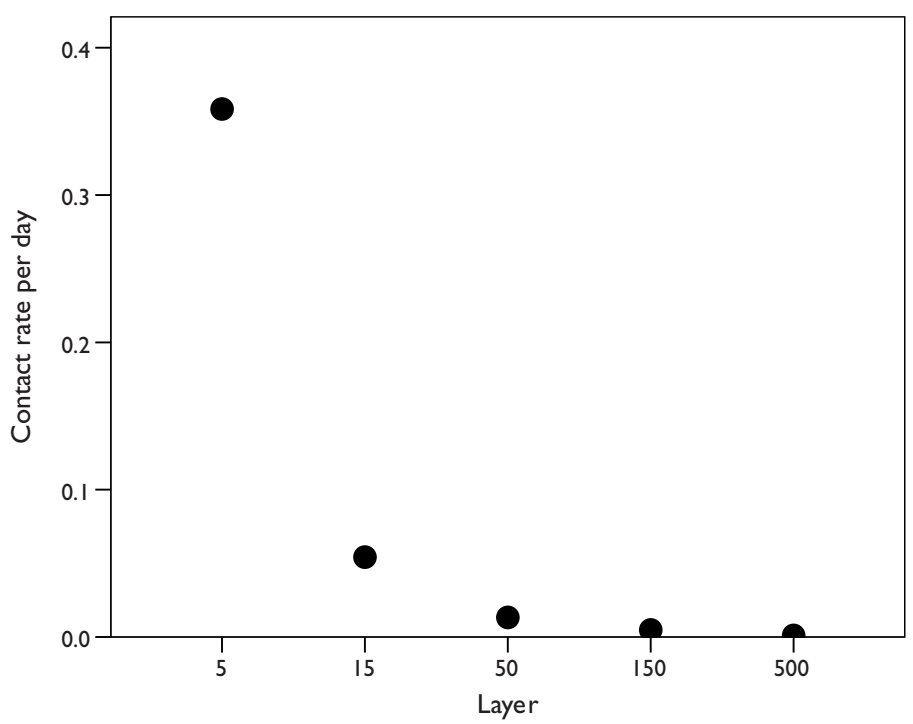

Figure 3. Mean time spent interacting with individual Alters in each layer of the network. Source: as for Figure 2.

\section{Trade-offs and constraints}

Primates bond their social groups through social grooming, and the time devoted to grooming is a linear function of group size (Dunbar, 1991; Lehmann et al., 2007). If humans were to bond their groups using the same mechanism, the time cost would be exorbitant (Dunbar, 1993). Instead, humans seem to have made social interaction more efficient by the acquisition of language to transform grooming into gossip (Dunbar, 1993; 1996). Nonetheless, because fitness is still driven by the basic need to feed and find food, there will always be an upper bound on the amount of time that any species can afford to devote to social interaction (Dunbar, 1993; Dunbar, Korstjens, \& Lehmann, 2009). Within the limits that this sets on the social time budget, each individual can choose to distribute its social time equitably or not. But if there is a tendency to 'favour the few' and distribute social time to form and maintain a small number of close relationships, then why should not all social time be devoted to core close relationships to the exclusion of all others? Clearly this is not the case, since nearly all studies of human friendship and social relationships report at least two bands of intensity (strong vs. weak ties: Granovetter, 1973; 1985; close vs. casual relationships: Hays, 1989; Wellman \& Wortley, 1990; core vs. peripheral ties: McPherson, Smith-Lovin, \& Brashears, 2006). More importantly, the data from our sample of $\sim 300$ complete personal social networks clearly demonstrate that humans invest much more time and effort in some relationships (especially those in the inner core of five intimates) than in others, with frequency of contact with Alters declining as a steep negative exponential function of network layer (Figure 3).

A more plausible suggestion, then, is that a cost-benefit mechanism limits the value of having an ever-increasing number of close ties in such a way as to naturally partition social relationships into at least two kinds: those in whom one invests a great deal of time and those with whom one spends less time, with knock-on consequences for the quality of the relationships concerned. In this, we follow both Brown and Brown (2006) in arguing that intimate relationships provide a level of emotional intensity that is crucial in creating close friendships, thereby facilitating the formation of close support 
coalitions and our own finding that frequency of contact with an Alter is positively correlated with self-rated emotional closeness to that individual (Hill \& Dunbar, 2003; Roberts et al., 2009). We extend Brown \& Brown's argument to differentiate between two distinct types of close relationships, namely the inner core of five intimates (often, but not necessarily, immediate family) and the next layer of around 10 close friends or family members. We suggest that the distinction between intimates and close friends lies in a balance in the distribution of costs and benefits with reference to their respective functional outcomes. Intimates identify a set of individuals who provide (and/or require) close emotional support that allows us to fine-tune emotional well-being and stress reduction as well as enabling us to interface with the wider social world. In this respect, they resemble the kinds of relationships identified in Fredrickson's (2004) 'broaden-andbuild' model of positive emotions. In this model, relationships exist to nurture positive emotions (which, as Fredrickson notes, have been largely ignored in psychology) that in turn provide a social and psychological environment within which individuals can not only survive better as individuals but also cooperate more effectively as functional groups. Such relationships allow us to reduce stress by their reassuring presence and, at least among adults, the promise of intervention in the conflicts by active support, as has been documented in primates (Crockford, Wittig, Whitten, Seyfarth, \& Cheney, 2008; Dunbar, 2010a; Wittig et al., 2008). Among humans, there are similarly striking effects of network membership on well-being: Fowler \& Christakis (2008), for example, have shown that the state of happiness of one's close friends, and even friends-of-friends, can prospectively influence one's own happiness. In contrast, the layer of close friends beyond this inner core of intimates provides a wider source of exchange at a personal level (reciprocal exchange of material goods and/or time [in the sense of 'helping out'], childcare, social activity, mutual protection against harassment, etc.).

Even though large support groups would seem to be more effective than smaller ones, leading to a selection pressure favouring ever larger coalitions, there must inevitably be a trade-off between the benefits that accrue and the cost of maintaining the relationships concerned. This is likely to be so for three reasons. First, only so many individuals may be able to provide the benefit at any one time (a too-many-cooks-spoil-the-broth effect). Research in the social psychology of group work confirms that benefits are asymptotic for groups with tasks that require close coordination. Larger task groups commonly fail to perform in proportion to their size (Steiner, 1972) for a number of reasons, including free riding and loss of motivation (Kerr, 1983), as well as process losses (e.g., production blocking in group brainstorming: Diehl \& Stroebe, 1991). Second, close relationships are usually reciprocal (Brown \& Brown, 2006), so while each relationship accumulates potential benefit to Ego, it does so at the cost of exposing Ego to the risk of being called upon to reciprocate commitments to all of the Alters with whom such a relationship has been built up. However, the real underlying basis of the trade-off is that, if the quality of a relationship (and hence its reliability) is a function of the time invested in it, there will be costs to creating and, in particular, maintaining relationships over time. In contrast to the benefits (which are likely to be asymptotic), these costs increase linearly with the number of relationships involved, since each relationship requires the investment of an equal amount of time and effort. If each relationship requires a minimum level of time commitment to maintain its quality (relationships decay over time if the members do not interact: see Canary \& Dainton, 2003; Hays, 1985; Roberts \& Dunbar, in press), every additional Alter means that an Ego's social time requirement must increase proportionately. 


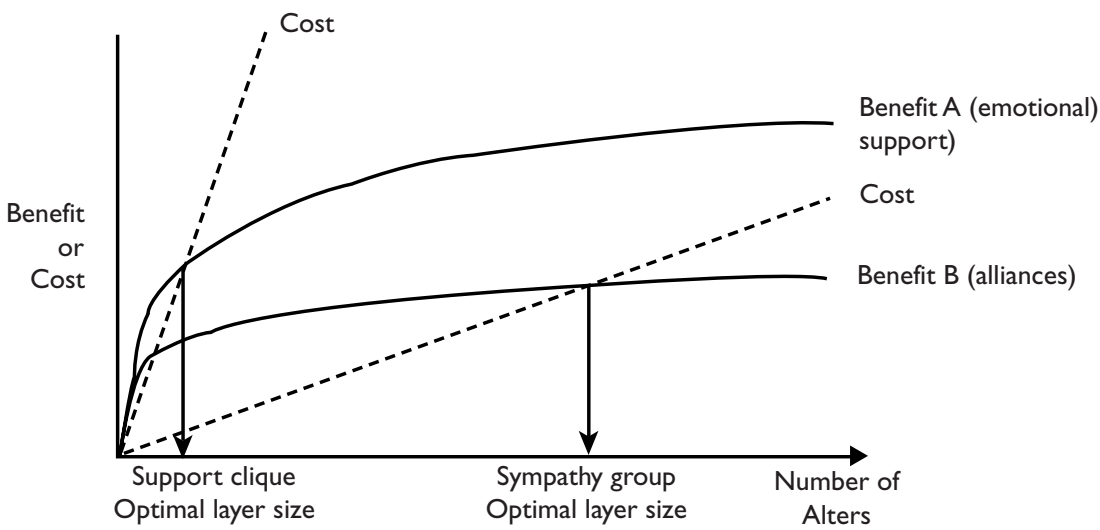

Figure 4. An optimality model of the trade-off between the (linear) time investment costs and the (asymptotic) benefits that accrue for relationships of different quality. Optimal numbers of Alters for a given relationship type are identified by the relevant Nash equilibrium (defined by the point where the relevant cost line intersects with the relevant benefit line). We assume that costs rise more or less linearly with the number of relationships that have to be maintained, and do so more steeply for relationships that require more intense relationships (high- vs. low-costs lines), while the benefits themselves are asymptotic (there is a limit to which adding more Alters will increase the benefit that accrues to Ego, with high vs. low benefits representing functionally different qualities of benefit such as emotional support vs. exchange of favours). The example considers only the two innermost network layers (support clique and sympathy group), but a similar argument can be made for the two outer layers of the network (affinity group and active network).

Ultimately, this process encounters a pair of insurmountable constraints: one is that there are only so many hours available in the day, the second being the fact that time is not elastic (i.e., compressible). The magnitude of this constraint is obvious from the time costs involved. If we assume that the average interaction lasts $30 \mathrm{~min}$ (see Eagle, Pentland, \& Lazar, 2009) and sum across the numbers in each layer multiplied by the contact rates given in Figure 3, the result is $2.25 \mathrm{~h}$ a day spent in social interaction, a figure that is comfortably within the average of $3.5 \mathrm{~h}$ a day that diary and time budget studies from a diverse sample of cultures indicates is actually spent in genuine social interaction (i.e., including casual interactions) (Dunbar, 1998b). If however, if each relationship in every layer required the same intensity of interaction as that lavished on support clique members (as shown in Figure 3), the total required daily socializing budget would be an impossible $39.4 \mathrm{~h}$ a day.

The issue, then, is what number of intimates optimizes net benefit by maximizing the gain while minimizing the costs. The trade-off is between spreading oneself thinly to gain many potential allies, and concentrating social time more conservatively to build a higher level of trust among a smaller number of allies. Social support and the risk trade-off might thus explain the SBH observation that people tend to have a small number of very close friends, a slightly larger number of good friends and a large number of acquaintances.

We can model this as a standard optimality problem (Figure 4). While there is a common cost baseline (the unitary time cost of maintaining individual friendships), this is modulated by how intense the process of relationship formation is. In other words, really intense relationships require relatively more time investment, yielding a steeper cost curve (contrast the high- vs. low-cost curves in Figure 4). Benefits also increase with 
the number of Alters offering a particular resource type, but, as is invariably the case with benefit curves, reach an asymptote as a result of a satiation effect (in part a consequence of the limitations on the marginal gains we discussed above). Some resource types (e.g., emotional support) have high fitness valency, and thus asymptote at an absolutely higher level, but reach that asymptote earlier because they impose comparatively high demands; in contrast, other resources (generalized exchange relationships for childcare and other reciprocated benefits) rise to a lower asymptote more slowly because 'too many cooks' effects are less intrusive and/or a minimum pool of potential allies is needed to guarantee the benefit (e.g., to find someone with the time to spare to help out). In each case, the Nash equilibrium (which defines the optimal solution) is given by the intersection of the respective cost and benefit lines. This yields two separate optimal group sizes, a smaller number of more intensely rewarding and demanding relationships and a larger number of less intense ones, as illustrated in Figure 4.

Although the outer layers at 50 (affinity group) and 150 (active network) are not equally well understood, we can nonetheless conjecture something about the functions of these layers. We know from our network data that these layers consist disproportionately of extended family members (Roberts et al., 2009), who are bound to Ego through obligate ties of kinship that have a significant impact on willingness to act altruistically (Curry, Roberts, \& Dunbar, under revision). One advantage of ties with extended kin over non-kin is that such ties are structural and hence persist in the absence of active maintenance (Roberts, 2010). While demands for assistance by or from kin are harder to turn down, the social investment in these ties between episodes of support may be minimal.

In ethnographic terms, the 50-layer for group size maps to the overnight camp (or band) size in traditional small-scale human societies (Dunbar, 1993). This grouping level provides a larger number of individuals for different activities divided between male (hunter) and female (gatherer) roles, and might also be related to protection against predators (as we noted above, the main driver for group size evolution among primates). Both sexes tend to form relationships in age-related cohorts, so a 50-band may be a consequence of coalescing three sympathy groups for overnight predator protection (the period during which predation risk is much the highest for humans), with the added benefit of information sharing as well as cooperative foraging. Beyond the typical group size, however, increased coordination requirements are likely to lead to process losses such that benefits again asymptote. Overnight camp groups depend on much weaker ties (Figure 3) linking families or other clusters. As a result, these larger groups are relatively unstable in hunter-gatherer societies, and tend to change membership on a scale of months as individual families come and go due to diverging interests and/or petty squabbles. In contemporary terms, we might envisage the 50-layer providing for a wider range of willing exchange partners, but ones that are articulated on a more strictly reciprocated basis (help is only given when repayment is reasonably certain).

The 150-level grouping (the community or clan in the ethnographic literature) may be important for inter-group conflict in traditional societies, where larger groups are especially important because sheer size matters. This layer is composed of individuals who are socially familiar with most other members of the larger collective, with clusters linked together in a 'small world' structure. The embedded layers of social relationships supplemented by weak ties that connect all clusters (without connecting all individuals) may provide sufficient cohesion for effective collective action in inter-community conflict (leading to classic in-group/out-group effects). Furthermore, considering that the younger male cohort in the group may only number one-eighth or so of the whole 
community, most males are likely to be defending their community with individuals with whom they have reasonably intense (support, sympathy clique) relationships. In traditional societies, this is often engineered by creating age cohorts who become intensely bonded with each other during what are often deliberately frightening group puberty rituals.

Another driver for forming weak ties in this outer layer may be information exchange and access to resources. The more people an individual knows and socializes with, the better that individual's information flow (sensu Granovetter, 1973), and hence the better their survival intelligence for finding food or avoiding local environmental problems. It may also provide a pool of recruits with whom to form more intense relationships should the need arise (e.g., through death, broken friendships, etc.). Little social interaction time is needed per individual for relationship maintenance at this level (Figure 3), but there are many individuals to interact with. In contemporary post-industrial societies, this information exchange function may now be the most important function of this outer layer. Opportunities for mate choice might also be an important consideration at this social level, as has been explicitly argued by Caporael and Baron (1997). Christakis and Fowler (2010) note that around $75 \%$ of introductions to future life partners come through the first three steps in the social network (i.e., the friends of friends of friends), which is probably equivalent to the $\mathbf{1 5 0}$ layer of egocentric networks.

It is worth emphasizing here that the selection pressures driving the emergence of a particular layer do not limit the functionality of that layer; rather, selection acts on the psychological mechanisms that enable a group of a particular size to be realized (and so have the desired functional outcome). In contemporary terms, this means that we have natural cognitive propensities for groups of a particular size and relationship quality, not for a particular function. Consequently, groupings represented by the different layers can be used for any functional purpose for which relationships of a particular quality or intensity defined by that layer are a design feature. In other words, work group sizes are task specific. If a task requires intense relationships and deep social knowledge (i.e., understanding of individual members' behavioural idiosyncrasies) between its members, then there will be a limit on the size of work group that can handle that kind of task efficiently to the innermost layer of five intimates. Conversely, tasks that require larger numbers of individuals (e.g., brainstorming) may obviate the possibility of dealing effectively with tasks that require more intimate personal knowledge. Within these constraints, however, we can adapt our psychological mechanisms to deal with any task that has the defining psychological design characteristics in a horses-for-courses fashion.

\section{Trust in social relationships}

The maintenance of coherent, long-lasting relationships seems likely to depend on at least two key constraints: the time required to establish and maintain a relationship and the cognitive demands of maintaining a functional mental database that can be used to manipulate information about one's social world (Dunbar, 2008). This emphasis on constraints is in line with social interdependence theory (Kelley et al., 1983; Thibaut \& Kelley, 1959), which normally applies to non-kin. Investment in kin offers, of course, a direct fitness benefit that requires no further explanation in terms of social benefits: in evolutionary terms, altruism towards kin can be explained in terms of the theory of kin selection (i.e., Ego gains in inclusive fitness by assisting relatives who share his/her genes to reproduce more effectively: Hamilton, 1964). Although other constraints are 
doubtless operating as well, we emphasize these two core components because they bridge into the non-human social world as well (Dunbar \& Shultz, 2010).

Many of the relationships we have discussed involve reciprocation of commitment or exchange relationships among non-kin that necessarily rely on trust - trust that (1) other members of one's network will cooperate or reciprocate when required to do so and (2) none will deliberately undermine or abuse that relationship (Dunbar, 1998a). In this section, we elaborate on a trust mechanism that gives us a psychological understanding of why and how social time budgets might be distributed over types of relationship in the way they seem to be.

Most definitions relate trust to reciprocity and collaboration (Deutsch, 1985; Ostrom, 2002; Rotter, 1971), whereby repeated interaction between two individuals generates and affirms judgments that the other party is trustworthy. As defined by Rotter (1971), trust is 'an expectancy held by an individual or a group that the word, promise, verbal or written statement of another individual or group can be relied on'. Theories of human friendship (Brown \& Brown, 2006; Hays, 1985; Oswald et al., 2004) note the importance of reciprocity and exchange of benevolent acts in building social relationships and, implicitly, trust. Empirical studies of friendship (Hays, 1985, 1989; Oswald et al., 2004) point to the advantage of investing in fewer, more intimate relationships. Furthermore, the close correlation between interaction frequency and relationship intimacy (Hill \& Dunbar, 2003) supports the claim that trust formation for intimate relationships requires considerable investment in social interaction (Roberts \& Dunbar, in press).

Mutual trust supports mutual reliance on one another for help, emotional support and companionship (Baumeister \& Leary, 1995). McCabe (2002) argues that trust evolved to enhance collaboration through improved abilities to assess trustworthiness and detect deceit in social interactions. This depends on the human theory of mind capacity to reflect on the social intentions of others. Communication increases cooperation and trust in social dilemma games (Cook \& Cooper, 2002; Ostrom, 2002) and, as more evidence accrues through repeated interaction, trust may change from 'calculative mode' (in which an individual's trustworthiness is assessed on limited evidence) to relational trust through an emotional response based on direct social experience. Several authors (Fesslet \& Haley, 2003; Planalp, Fitness, \& Fehr, 2006; Silk, 2003) have argued that the role of emotion in friendship is to change detailed calculation of costs and benefits, exemplified in tit-for-tat simulations of cooperation (Roberts \& Renwick, 2003), into bonds that are less susceptible to adverse interactions.

We propose that trust not only facilitates dyadic and group-level collaboration but also underpins the formation and maintenance of social relationships. Trust may give rise to distinct relationship layers via an affect component that reinforces attachment in close relationships. If individuals socially 'groom' each other frequently over an extended period of time, the level of mutual trust should increase to the stage where emotion (i.e., pleasure in the Alter's company) becomes more important than the rewards of collaboration per se. This emotional engagement then provides the basis for future commitment whenever this might be needed, and the level of pleasure in Alter's company can serve as an index of how much they can be relied on. The degree of trust is therefore closely related to emotional closeness; however, as a relationship becomes closer, affect reduces the need to consciously calculate trust, and hence alleviates the cognitively costly theory of mind burden (Powell, Lewis, Dunbar, García-Fiñana, \& Roberts, 2010) in tracking very close (support group) relationships. However, the influence of affect on trust may be less appropriate for the sympathy clique relationships where formation of alliances may necessitate a more conscious calculation of trust. 




Figure 5. Schematic outline of how trust develops over time, stabilizes out, and responds to challenges for relationships corresponding to the three main network layers (strong, medium, and weak ties, notionally corresponding to the 5,15 , and 150 layers, respectively). The model assumes that increasing frequency of interaction results in increased levels of trust. However, although trust rises (perhaps linearly) as a function of the frequency of interaction, there are a series of levels that trust naturally stabilizes at (represented by the plateaux); trust can rise above these stable points only if significant additional social effort is invested in the relationship. These stable points correspond to the different kinds of relationships found in the various network layers. The $X$ symbols indicate events that destabilize relationships (insults, sins of omission or commission, etc.). Weak ties are relatively immune to such insults due to their low trust level. However, medium ties are more sensitive, and exhibit an immediate decline in trust following an insult, after which trust may rebuild. If too many insults occur together, however, a medium tie might decline precipitously and become a weak tie. At the highest level of trust, an affect buffer comes into play and buffers the tie against isolated negative events.

The interaction between trust and relationships is summarized in Figure 5, showing three levels of trust formation, each respectively, corresponding to a different relationship layer. As a general rule, trust develops over time as dyads interact, settling at a particular trust (i.e., relationship) level if interaction rates are not frequent enough to lift them above the threshold required for a higher layer. Strong ties exhibit more resilience to unfriendly interactions, giving intimate friends the benefit of the doubt, whereas sympathy group relationships will be more susceptible to variation in trust in response to unfriendly interactions, reflecting a conscious, calculative mode. Of course, kin relationships may follow a different model, since they seem to show more resilience to low maintenance (Roberts \& Dunbar, in press). Strong ties with non-kin in the inner circle of around 5 take longer to develop and have to rise to a higher level of trust than medium or weak ties (i.e., those corresponding to the 50 and 150 outer layers).

Interaction frequencies are one of the most reliable correlates of relationship strength in both humans and animals (Dunbar \& Shultz, 2010; Hays, 1989). For example, Hill and Dunbar (2003) showed that the level of emotional intimacy in a relationship correlates with the amount of time invested in it (see also Roberts \& Dunbar, in press), and Curry et al. (2011) have shown that emotional closeness reliably predicts willingness to act altruistically towards non-kin Alters.

\section{Affective and cognitive correlates of tie strength}

Linking the observed regularities of layers with distinct differences in psychological processes and responses is a task that we are only just starting to tackle. Meeting intimate friends should produce a measurably more intense emotional response than meeting just good friends, for example. Yet few studies have assessed emotional response to meeting friends, either via physiological measures [Galvanic Skin Response (GSR)], heart 
rate, pupilometry: Insel \& Fernald, 2004) or via affect questionnaires. The reward for intimate relationships might be mediated by emotional well-being and endorphin release (Dunbar, 2010c; Keverne, Martensz, \& Tuite, 1989; Machin \& Dunbar, in press), but so far neuropsychologists have not explored this possibility. Similarly, memory schemata for Alters in strong-tie relationships should be more detailed than those for mediumtie Alters in terms of intimate details, both about the other person and about their relationships with third parties. Relationship memory at the sympathy group level may more typically involve episodic recording of incidents in social history, for example. Again, these cognitive aspects of relationships have yet to be explored.

As would be expected by the steeper investment in the closest circles of support clique and sympathy group, stronger ties do tend to be more stable over time (e.g., Ledbetter, Griffin, \& Sparks, 2007), whereas weaker ties are more liable to change. In a study of late adolescents, for example, Roberts and Dunbar (in press) found that only $29.7 \%$ of kin Alters (a strong tie) moved from the inner layers (5-15 layers) to the outer layers (50-150 layers) over an 18-month period, compared to $51.4 \%$ of non-kin (i.e., friend) Alters (representing weaker ties). Stronger ties should also show more connectivity and centrality within the Ego network, especially within the support clique where inter-relationships between all the members may be particularly dense and interconnected (Roberts, 2010). Curry and Dunbar (2011) have shown that, within these inner network layers, altruism and emotional closeness towards friends is a function of network density (i.e., the extent to which one's friends are also friends with each other). Sympathy group relationships should be more fluid and reflect individuals' different interests beyond the cluster; more broadly, relationships will become increasingly diffuse and less well integrated as one moves out through the layers.

\section{Discussion}

We have suggested that patterns in social relationships evolved from simple behavioural predispositions, such as the tendency for individuals to distribute their social time budgets unevenly, thereby favouring formation of a small number of intense relationships while also devoting some of the social time budget to weaker ties. To explain the observed distributions of relationships at different levels of intensities, we posit that a set of cost-benefit trade-offs governs the distribution of individuals' social investment in others, and that the cost-benefit profile differs for different layers. These trade-offs, combined with constraints, which may also shape the levels of relationship intensities, give rise to relationship types that define the support, sympathy, and other levels of personal social networks. One underlying mechanism that governs the allocation of social investment in different layers may be the trust formation process, which creates an 'affect buffer' that makes strong ties less susceptible to decay. This affect buffer might function to free up more social time for Ego to invest in other relationships.

Relationship types or 'signatures' (i.e., consistent, recognizable signs in the form of behaviour) for support and sympathy groups point towards several potential empirical studies. Dyads of participants with existing relationships, pre-assessed using emotional closeness scales to assign them to support or sympathy groups, could be tested with scenario experiments to evaluate hypotheses about different types of support offered by each network layer. We predict that support group Alters would volunteer more demanding help more rapidly than sympathy group Alters, and these in turn more rapidly than Alters in the outer (50 and 150) layers. The quality of memory for Alters and their activities should also differ between the layers: memory of others in support relationships 
should be more detailed and up to date than those in sympathy relationships, whereas memory for sympathy group Alters should be more episodic and record critical incidents in coalitions and social conflicts. Surveys and ethnographic studies could illuminate how people manage their social time budget in different cultural groups, and test the hypothesis that the layers should be manifest in individuals' preferences for spending more time with a few (the support layer) significant Alters and less time with a larger number of friends (the sympathy layer), dividing the remainder of their social time among weak ties. Longitudinal studies on social time budgets may also test the resilience of different tie strengths to the lack of maintenance or other social stresses. In partial support of this, we have found that both strong and medium friendship (but not kinship) ties wane if they are not regularly serviced during the transition from school to university (Roberts \& Dunbar, in press).

A challenge for future studies will be to establish the strength of possible friendship signatures. The strong version of our signatures hypothesis should be manifest in discrete step functions in the distributions of emotional closeness and other friendship measures (e.g., Oswald et al., 2004); a weaker version of the hypothesis might be satisfied by triangulation of evidence (i.e., several measures generally point to the distinction between the support and sympathy layers, although no discrete change is apparent in any one measure).

The arguments advanced in this paper have been set within an egocentric network perspective; however, the relationship levels linked to the $\mathrm{SBH}$ raise questions as to how the Ego-level support and sympathy groups are integrated into top-down communitylevel network structures. While dense integration in which Ego and their Alters share support and sympathy friends with each other is possible, empirical evidence suggests that a more plausible match to actual social structure at the sympathy group level may be a 'hub and spokes' model in which support groups behave as densely connected cliques and sympathy-level relationships form the bridging spokes between support cliques (Roberts, 2010). Studies on the structure of social networks, for example, indicate a pattern of some dense clusters with overall high levels of interconnectivity (Hogan, 2008; Wellman et al., 2006), although data on relationship strength is difficult to equate with relationship types.

It is currently unclear to what extent network layers reflect group size levels that are causally based on the functional demands of particular tasks and constrained by the challenges of group dynamics, or whether they are, in a more explicitly hard-wired sense, derivative of cognition that was itself an adaptation to task demands in our evolutionary past. In other words, does human behaviour now fit social and work groups into the limits set by fundamental cognitive and physical constraints? The influence of computer technology on social relationships is a useful ecological probe into these conjectures. Because computer-mediated communication (CMC) such as e-mail, social networking sites, and texting mitigates the constraints of time and distance, it might be expected to ameliorate the constraints imposed by social time budgets by making communication more efficient. Interestingly, the majority of studies to date indicate that the major function of CMC has been to supplement the maintenance of strong and medium-strength relationships formed in the real world. There is scant evidence of any capacity increases in relationship numbers (Binder, Howes, \& Sutcliffe, 2009; Ellison et al., 2007; Lampe, Ellison, \& Steinfield, 2006; Pollett et al., 2011; Wellman, Haase, Witte, \& Hampton, 2001). Social networking sites do afford maintenance of a larger number of weak ties in friends lists (Donath \& Boyd, 2004; Joinson, 2008; Lampe et al., 2006); however, there is little evidence to suggest that many of these extra relationships are active in the social sense 
of Granovetter's $(1973,1985)$ weak ties or the affinity group. If anything, both the online debate on numbers of friends and surveys of actual friends' listings on individual sites strongly conform to the predictions of the social brain model.

One of our motivations in writing this paper was to investigate the convergence of theory in evolutionary, cognitive, and social psychology. The extension to SBH of relationship signatures and the trust mechanism leaves open at least three alternative deep causal explanations. First, relationships and, inter alia, trust formation may reflect constraints imposed by social cognitive capacities such as theory of mind (Stiller \& Dunbar, 2007) in that memory for managing relationships is limited not only by absolute capacity but also by accuracy and recency. Trust formation is also limited by a dilution effect as implied by a generalized Balance Theory (Cartwright \& Harary, 1956; Heider, 1946): if Ego spreads attention too thinly, the existing Alters feel betrayed by attention being devoted to others. A second explanation may be that relationships develop in different layers for other social motivations, such as the need for belonging and security during childhood and adolescence, and the capacity to form new deep relationships becomes exhausted by early adulthood. There is some evidence for this view in longitudinal studies of friendship (Hays, 1985; Grossetti, 2005), indicating that we form most strong ties by early adulthood. Third, it may be that our behaviour is at least partially determined by evolutionary echoes of our past in which selection pressure produced behavioural predispositions to partition social time budgets in an asymmetrical manner to favour a few deeper relationships at the expense of many weaker ones. The cost-benefit trade-offs could link to behaviour that enhances fitness and survival at the individual level, in the form of improved survival of offspring and parents through reciprocal social support. Investigating the relative contribution of these mechanisms is a future research challenge in theory development and will require empirical studies to tease apart complex interacting influences on human social network evolution and maintenance.

\section{Acknowledgements}

The preparation of this paper was supported by ESRC/EPSRC Cognitive Foresight Programme project TESS (Developing Theory for Evolving Socio-technical Systems). RD's contribution was also supported by the British Academy Centenary Project ('Lucy to Language') and the EU-FP7 'SocialNets' and 'ICTe-collective' Projects.

\section{References}

Alexander, R. D. (1974). The evolution of social behavior. Annual Review of Ecology and Systematics, 5, 352-383.

Andersen, S. M., \& Chen, S. (2002). The relational self: An interpersonal social-cognitive theory. Psychological Review, 109, 619-645.

Baumeister R. F., \& Leary, M. R. (1995). The need to belong: Desire for interpersonal attachments as a fundamental human motivation. Psychological Bulletin, 117, 497-529.

Berscheid, E. (1994). Interpersonal relationships. Annual Review of Psychology, 45, 79-129.

Binder, J., Howes, A., \& Sutcliffe, A. G. (2009). The problem of conflicting social spheres: Effects of network structure on experienced tension in social network sites. Proceedings: CHI '09 human factors in computing systems, Boston. New York: ACM Press.

Brewer, M., \& Gardner, W. (1996). Who is this 'We'? Levels of collective identity and self representations. Journal of Personality and Social Psychology, 71, 83-93.

Brown, S. L., \& Brown, R. M. (2006). Selective investment theory: Recasting the functional significance of close relationships. Psychological Inquiry, 17, 1-29.

Buys, C. J., \& Larson, K. L. (1979). Human sympathy groups. Psychology Reports, 45, 547-553. 
Canary, D. J., \& Dainton, M. (2003). Maintaining relationships. In A. Vangelisti \& D. Perlman (Eds.), The Cambridge handbook of personal relationships. New York: Cambridge University Press.

Caporael, L. R., \& Baron, R. M. (1997). Groups as the mind's natural environment. In J. A. Simpson \& D. T. Kenrick (Eds.), Evolutionary social psychology. Mahwah, NJ: Erlbaum Associates.

Cartwright, D., \& Harary, F. (1956). Structural balance: A generalization of Heider's theory. Psychological Review, 63, 277-293.

Christakis, N., \& Fowler, J. (2010). Connected: The surprising power of our social networks. New York: Harper \& Collins

Cohen, S. (2004). Social relationships and health. American Psychologist, 59, 676-684.

Cohen, S., \& Wills, T. A. (1985). Stress, social support, and the buffering hypothesis. Psychological Bulletin, 98, 310-357.

Cook, K. S., \& Cooper, R. M. (2002). Experimental studies of cooperation, trust and social exchange. In E. Ostrom \& J. Walker (Eds.), Trust and reciprocity: Interdisciplinary lessons from experimental research (pp. 209-244). New York: Russel Sage Foundation.

Crockford, C., Wittig, R. M., Whitten, P. L., Seyfarth, R. M., \& Cheney, D. L. (2008) Social stressors and coping mechanisms in wild female baboons (Papio hamadryas ursinus). Hormones and Bebavior, 53, 254-265.

Curry, O., \& Dunbar, R. I. M. (2011). Altruism in networks: The effect of connections. Biology Letters. Advance online publication. doi:10.1098/rsbl.2010.1202

Curry, O., Roberts, S. B. G., \& Dunbar, R. I. M. (2011). The strength of ties in social networks: Altruism is more closely related to emotional closeness among friends than among family. Manuscript submitted for publication.

Dainton, M., Zelley, E., \& Langan, E. (2003). Maintaining friendships throughout the lifespan. In D. J. Canary \& M. Dainton (Eds.), Maintaining relationships through communication. Mahwah NJ: Lawrence Erlbaum Associates.

Deutsch, M. (1985). The resolution of conflict: Constructive and destructive processes. New Haven: Yale University Press.

Diehl, M., \& Stroebe, W. (1991). Productivity loss in idea-generating groups: Tracking down the blocking effect. Journal of Personality and Social Psychology, 61, 392-403.

Donath, J., \& Boyd, D. (2004). Public displays of connection. BT Technology Journal, 22, 71-82.

Dunbar, R. I. M. (1988). Primate social systems. London: Chapman \& Hall.

Dunbar, R. I. M. (1991). Functional significance of social grooming in primates. Folia Primatologica, 57, 121-131.

Dunbar, R. I. M. (1992). Neocortex size as a constraint on group size in primates. Journal of Human Evolution, 22, 469-493.

Dunbar, R. I. M. (1993). Coevolution of neocortex size, group size and language in humans. Behavioral Brain Sciences, 16, 681-735.

Dunbar, R. I. M. (1996). Grooming, gossip and the evolution of language. London: Faber \& Faber.

Dunbar, R. I. M. (1998a). The social brain hypothesis. Evolutionary Antbropology, 6, 178-190.

Dunbar, R. I. M. (1998b). Theory of mind and the evolution of language. In J. R. Hurford, M. Studdart-Kennedy, \& C. Knight (Eds.), Approaches to the evolution of language, Cambridge: Cambridge University Press.

Dunbar, R. I. M. (2008). Mind the gap: Or why humans aren't just great apes. Proceedings British Academy, 154, 403-423.

Dunbar, R. I. M. (2010a). Brain and behaviour in primate evolution. In P. H. Kappeler \& J. Silk (Eds.) Mind the gap: Tracing the origins of buman universals (pp. 315-330). Berlin: Springer.

Dunbar, R. I. M. (2010b). Constraints on the evolution of social institutions and their implications for information flow. Journal of Institutional Economics. Advance online publication. doi:10. 1017/S1744137410000366

Dunbar, R. I. M. (2010c). The social role of touch in humans and primates: Behavioural function and neurobiological mechanism. Neuroscience and Biobebavioural Reviews, 34, 260-268.

Dunbar, R. I. M., Korstjens, A., \& Lehmann, J. (2009). Time as an ecological constraint. Biological Reviews, 84, 413-429. 
Dunbar, R. I. M., \& Shultz, S. (2007). Understanding primate brain evolution. Philosophical Transactions of the Royal Society of London, 362B, 649-658.

Dunbar, R. I. M., \& Shultz, S. (2010). Bondedness and sociality. Behaviour, 147, 775-803.

Dunbar, R. I. M., \& Spoors, M. (1995). Social networks, support cliques, and kinship. Human Nature, 6, 273-290.

Eagle, N., Pentland, A., \& Lazar, D. (2009). Inferring friendship network structure by using mobile phone data. Proceedings of the National Academy of Sciences of the United States of America, $106,15274-15278$.

Ellison, N. B., Steinfield, C., \& Lampe, C. (2007). The benefits of facebook "friends": Social capital and college students' use of online social network sites. Journal of Computer-Mediated Communication, 12, 1143-1168.

Fessler, D. M. T., \& Haley, K. J. (2003). The strategy of affect: Emotions in human cooperation. In P. Hammerstein (Ed.), Genetic and cultural evolution of cooperation (pp. 7-36). Cambridge, MA: MIT Press.

Fischer, C. S. (1982). To dwell among friends. Chicago: University of Chicago Press.

Fowler, J. J., \& Christakis, N. (2008). Dynamic spread of happiness in a large social network: Longitudinal analysis over 20 years in the Framingham Heart Study. British Medical Journal, 337, a2338. doi:10.1136/bmj.a2338

Fredrickson, B. L. (2004). The broaden-and-build theory of positive emotions. Philosophical Transactions of the Royal Society, London, 359B, 1367-1377.

Girvan, M., \& Newman, M. E. J. (2002). Community structure in social and biological networks. Proceedings of the National Academy of Sciences, 99, 7821-7826.

Golden, J., Conroy, R., \& Lawlor, B. (2009). Social support network structure in older people: Underlying dimensions and association with psychological and physical health. Psychology, Health \& Medicine, 14, 280-290.

Granovetter, M. (1973). The strength of weak ties. American Journal of Sociology, 78, 1360-1380.

Granovetter, M. (1985). Economic action and social structure: The problem of embeddedness. American Journal of Sociology, 91, 481-510.

Grossetti, M. (2005). Where do social relations come from? A study of personal networks in the Toulouse area of France. Social Networks, 27, 289-300.

Grossetti., M. (2007). Are French networks different? Social Networks, 29, 391-404.

Hamilton, W. D. (1964). The genetical evolution of social behaviour. I, II. Journal of Theoretical Biology, 7, 1-52.

Hamilton, M. J., Milne, B. T., Walker, R. S., Burger, O., \& Brown, J. H. (2007). The complex structure of hunter-gatherer social networks. Proceedings of the Royal Society of London, 274, 2195-2202.

Hays, R. B. (1985). A longitudinal study of friendship development. Journal of Personality and Social Psychology, 48(4), 909-924.

Hays, R. B. (1989). The day-to-day functioning of close versus casual friendships. Journal of Social and Personal Relationships, 6, 21-37.

Heider, F. (1946). Attitudes and cognitive organization. Journal of Psychology, 21, 107-112.

Helgeson, V. (2003). Social support and quality of life. Quality of Life Research: An International Journal of Quality of Life Aspects of Treatment, Care \& Rehabilitation, 12 (Supplement 1), 25-31.

Hill, R. A., Bentley, A., \& Dunbar, R. I. M. (2008). Network scaling reveals consistent fractal pattern in hierarchical mammalian societies. Biological Letters, 4, 748-751.

Hill, R. A., \& Dunbar, R. I. M. (2003). Social network size in humans. Human Nature, 14, 53-72.

Hogan, B. (2008). Analyzing social networks via the Internet. In N. Fielding, R. Lee, \& G. Blank (Eds.), The handbook of online research methods. Thousand Oaks CA: Sage.

Insel, T. R., \& Fernald, R. D. (2004). How the brain processes social information: Searching for the social brain. Annual Review of Neuroscience, 27, 697-722.

Joinson, A. N. (2008). Looking at, looking up or keeping up with people? Motives and use of Facebook. Proceedings of the Twenty-sixth Annual SIGCHI Conference on Human Factors in Computing Systems, CHI-08. New York: ACM Press. 
Kayahara, J., Wellman, B., Boase, J., Hogan, B., \& Kennedy, T. (2005). Canadians, culture and computers: Report to Heritage Canada. Wellman Associates.

Kelley, H. H., Berscheid, E., Christensen, A., Harvey, J. H., Huston, T. L., Levinger, G.... Peterson, D. R. (1983). Close relationships. New York: Freeman.

Kelley, H. H., Holmes, J. G., Kerr, N. L., Reis, H. T., Rusbult, C. E., \& Van Lange, P. A. M. (2003). An atlas of interpersonal situations. Cambridge: Cambridge University Press.

Kerr, N. L. (1983). Motivation losses in small groups: A social dilemma analysis. Journal of Personality and Social Psychology, 45, 819-828.

Keverne, E. B., Martensz, N., \& Tuite, B. (1989). Beta-endorphin concentrations in cerebrospinal fluid of monkeys are influenced by grooming relationships. Psychoneuroendocrinology, 14, 155-161.

Kraut, R., Kiesler, S., Boneva, B., Cummings, J., Helgeson, V., \& Crawford, A. (2002). Internet paradox revisited. Journal of Social Issues, 58, 49-74.

Kudo, H., \& Dunbar, R. I. M. (2001). Neocortex size and social network size in primates. Animal Behaviour, 62, 711-722.

Lampe, C., Ellison, N., \& Steinfield, C. A. (2006). A Face(book) in the crowd: Social searching vs. social browsing. Proceedings: CSCW-2006 (pp. 167-170). New York: ACM Press.

Ledbetter, A. M., Griffin, E., \& Sparks, G. G. (2007). Forecasting "friends forever": A longitudinal investigation of sustained closeness between best friends. Personal Relationships, 14, 343350.

Lehmann, J., Korstjens, A. H., \& Dunbar, R. I. M. (2007). Group size, grooming and social cohesion in primates. Animal Behaviour, 74, 1617-1629.

Machin, A. J., \& Dunbar, R. I. M. (in press). The brain opioid theory of social attachment: A review of the evidence. Behaviour.

Markus, H. R., \& Kitayama, S. (1991). Culture and the self: Implications for cognition, emotion, and motivation. Psychological Review, 98, 224-253.

McCabe, K. A. (2002). A cognitive theory of reciprocal exchange. In E. Ostrom \& J. Walker (Eds.), Trust and reciprocity: Interdisciplinary lessons from experimental research (pp. 147-169). New York: Russel Sage Foundation.

McPherson, M., Smith-Lovin, L., \& Brashears, M. E. (2006). Social isolation in America: Changes in core discussion networks over two decades. American Sociological Review, 71, 353-375.

Mesch, G., \& Talmud, I. (2007). Similarity and the quality of online and offline social relationships among adolescents in Israel. Journal of Research on Adolescence, 17, 455-466.

Milardo, R. M. (1992). Comparative methods for delineating social networks. Journal of Social and Personal Relationships, 9, 447-461.

Morahan-Martin, J., \& Schumacher, P. (2003). Loneliness and social uses of the Internet. Computers in Human Behavior, 19, 659-671.

Moreland, R. L., \& Levine, J. M. (1982). Socialization in small groups: Temporal changes in individual-group relations. In L. Berkowitz (Ed.), Advances in experimental social psychology (Vol. 15). New York: Academic Press.

Ostrom, E. (2002). Towards a behavioural theory linking trust, reciprocity and reputation. In E. Ostrom \& J. Walker (Eds.), Trust and reciprocity: Interdisciplinary lessons from experimental research (pp. 19-79). New York: Russel Sage Foundation.

Oswald, D. L., Clark, E. M., \& Kelly, C. M. (2004). Friendship maintenance: An analysis of individual and dyad behaviours. Journal of Social and Clinical Psychology, 23, 413-441.

Planalp, S., Fitness, J., \& Fehr, B. (2006). Emotion in theories of close relationships. In A. L. Vangelisti, \& D. Perlman (Eds.), The Cambridge handbook of personal relationships (pp. 369-384). Cambridge, UK: Cambridge University Press.

Pollett, T., Roberts, S., \& Dunbar, R. (2011). Use of social network sites and instant messaging does not lead to increased offline social network size, or to emotionally closer relationships with offline network members. Cyberpsychology, Behavior and Social Networking, 14, 253-258.

Powell, J., Lewis, P., Dunbar, R. I. M., García-Fiñana, M., \& Roberts, N. (2010). Orbital prefrontal cortex volume correlates with social cognitive competence. Neuropsychologia, 48, 35543562 . 
Roberts, G., \& Renwick, J. S. (2003). The development of cooperative relationships: An experiment. Proceedings of the Royal Society of London, 270B, 2279-2283.

Roberts, S. B. G. (2010). Constraints on social networks. In R. I. M. Dunbar, C. Gamble, \& J. A. J. Gowlett (Eds.), Social brain, distributed mind (pp. 117-138). Oxford: Oxford University Press.

Roberts, S. B. G., \& Dunbar, R. I. M. (in press). Communication in social networks: Effects of kinship, network size and emotional closeness. Personal Relationships.

Roberts, S. B. G., Dunbar, R. I. M., Pollet, T., \& Kuppens, T. (2009). Exploring variations in active network size: Constraints and Ego characteristics. Social Networks, 31, 138-146.

Rose, S., \& Serafica, F. (1986). Keeping and ending casual, close and best friendships. Journal of Social and Personal Relationships, 3, 275-288.

Rotter, J. B. (1971). Generalised expectancies for interpersonal trust. American Psychologist, 26, 443-452.

Rusbult, C. E., \& Van Lange, P. A. (2003). Interdependence, interaction, and relationships. Annual Review of Psychology, 54, 351-375.

Seeman, T., Lusignolo, T., Albert, M., \& Berkman, L. (2001). Social relationships, social support, and patterns of cognitive aging in healthy, high-functioning older adults: MacArthur Studies of Successful Aging. Health Psychology, 20, 243-255.

Shultz, S., \& Dunbar, R. I. M. (2007). The evolution of the social brain: Anthropoid primates contrast with other vertebrates. Proceedings of the Royal Society of London, 274B, 2429-2436.

Shultz, S., \& Dunbar, R. I. M. (2010). Encephalisation is not a universal macroevolutionary phenomenon in mammals but is associated with sociality. Proceedings of the National Academy of Sciences of the United States of America, 107, 21582-21586.

Shultz, S., Noe, R., McGraw, S., \& Dunbar, R. I. M. (2004). A community-level evaluation of the impact of prey behavioural and ecological characteristics on predator diet composition. Proceedings of the Royal Society of London, 271B, 725-732.

Silk, J. B. (2003). Cooperation without Counting: The puzzle of friendship. In P. Hammerstein (Ed.), Genetic and cultural evolution of cooperation (pp. 27-54). Cambridge MA: MIT Press.

Steiner, I. D. (1972). Group process and productivity. New York: Academic Press.

Stiller, J., \& Dunbar, R. I. M. (2007). Perspective-taking and social network size in humans. Social Networks, 29, 93-104.

Thibaut, J. W., \& Kelley, H. H. (1959). The social psychology of groups. New York: Wiley.

Van Schaik, C. P. (1983). Why are diurnal primates living in groups? Behaviour, 87, 120144.

Wellman, B., Carrington, P. J., \& Hall, A. (1988). Networks as personal communities. In B. Wellman \& S. D. Berkowitz (Eds.), Social structures: A network analysis (pp. 130-184). Cambridge: Cambridge University Press.

Wellman, B., Haase, A. Q., Witte, J., \& Hampton, K. (2001). Does the internet increase, decrease, or supplement social capital? American Behavioural Scientist, 45, 436-455.

Wellman, B., Hogan, B., Berg, K., Boase, J., Carrasco, J. A., Côté, R. . . . Tran, P. (2006). Connected lives: The project. In P. Purcell (Ed.), Networked neighborboods. Berlin: Springer.

Wellman, B., \& Wortley, S. (1990). Different strokes from different folks: Community ties and social support. American Journal of Sociology, 96, 558-588.

Wittig, R. M., Crockford, C., Lehmann, J., Whitten, P. L., Seyfarth, R. M., \& Cheney, D. L. (2008). Focused grooming networks and stress alleviation in wild female baboons. Hormones and Behavior, 54, 170-177.

Zhou, W., Sornette, D., Hill, R. A., \& Dunbar, R. (2005). Discrete hierarchical organization of social group sizes. Proceedings of the Royal Society of London, 272B, 439-444.

Received 28 September 20 10; revised version received I8 May 20II 